\title{
Activin A inhibits antigen-induced allergy in murine epicutaneous sensitization
}

\section{Magdalini Kypriotou ${ }^{1}$, Dianelys Rivero ${ }^{1}$, Sergio Haller ${ }^{2}$, Anita Mariotto ${ }^{1}$, Marcel Huber $^{1}$, Hans Acha-Orbea ${ }^{2}$, Sabine Werner ${ }^{3}$ and Daniel Hohl ${ }^{1}$ *}

1 Laboratory of Cutaneous Biology, Service of Dermatology and Venereology, Beaumont Hospital, CHUV, Lausanne, Switzerland

2 Department of Biochemistry, University of Lausanne, Lausanne, Switzerland

${ }^{3}$ Department of Biology, Institute of Molecular Health Sciences, ETH Zurich, Zurich, Switzerland

\section{Edited by:}

Heiko Mühl, Goethe University

Frankfurt, Germany

Reviewed by:

Ralf J. Ludwig, University of Lübeck, Germany

Miriam Wittmann, University of Leeds, UK

George Bougharios, University of

Oxford, UK

*Correspondence:

Daniel Hohl, Service of Dermatology and Venereology, Beaumont Hospital, CHUV, 04-437, Beaumont Avenue 29, 1011 Lausanne, Switzerland e-mail:daniel.hohl@chuv.ch
Activin A, a member of the TGF $\beta$ superfamily, is involved in physiological processes such as cell differentiation, tissue homeostasis, wound healing, reproduction, and in pathological conditions, such as fibrosis, cancer, and asthma. Activin enhances mast cell maturation, as well as regulatory T-cell and Langerhans cell differentiation. In this study we investigated the potential role of activin in epicutaneous sensitization with ovalbumin (OVA), notably with respect to its effect on known Th2-polarization. For this purpose, transgenic mice overexpressing activin in keratinocytes and their wild-type (WT) controls were sensitized epicutaneously with OVA. Skin biopsies were analyzed with regard to histopathological features and mRNA expression of pro-inflammatory andTh1/Th2 cytokines, and Ig levels were measured in the serum. Unexpectedly, activin overexpressing animals were protected from Th2-cytokine expression and induction of OVA-specific lgE levels compared to WT animals. On the other hand, transgenic mice were more susceptible to inflammation compared to WT littermates after tape-stripping and saline (vehicle) or OVA application, as shown by increased pro-inflammatory cytokine mRNA levels and neutrophil accumulation at the site of the treatment. We conclude that activin protects from antigen-induced cutaneous Th2polarization through modulation of the immune response. These findings highlight the role of activin in cutaneous sensitization, allergy, and in skin homeostasis.

Keywords: activin, epicutaneous sensitization, transgenic mice, inflammation, atopy

\section{INTRODUCTION}

Activins belong to the "transforming growth factor" (TGF $\beta$ ) superfamily of cytokines and growth factors. The most abundant activin variant is activin $\mathrm{A}$, the homodimer formed by two $\beta \mathrm{A}$ subunits connected by a disulfide bridge $(1,2)$. In addition to the well-characterized dimers, activin $\mathrm{A}(\beta \mathrm{A} \beta \mathrm{A}), \mathrm{B}(\beta \mathrm{B} \beta \mathrm{B})$, and $\mathrm{AB}(\beta \mathrm{A} \beta \mathrm{B})$, two additional $\beta$ chains $(\beta C$ and $\beta E$ ) have been described. Activins exert their biological effects through activation of transmembrane serine/threonine kinase receptors. Binding to a type II activin receptor (ActRIIA or ActRIIB) leads to the recruitment, phosphorylation, and activation of a type I activin receptor $($ ActRIB $=$ Alk 4 , or ActRIC $=$ Alk7 $)$. This activates the canonical signaling pathway via Smad proteins (Smad2/3/4; C. Elegans SMA and Mothers against decapentaplegic homologs), or, alternatively, mitogen-activated kinase pathways (MAPK). Activin bioavailability is regulated by natural soluble inhibitors, such as follistatin, follistatin-related protein (FRP), and inhibin, and by membrane-bound proteins, such as betaglycan (in complex with inhibin), crypto, and BAMBI (3). Activins were initially described as reproductive hormones, but they also have important functions in development, tissue homeostasis, and repair. Activins enhance fibrosis and epidermal skin cancer, promote bone and skin wound repair, and act as pro- or anti-inflammatory proteins in a cell type and organ dependent manner. Thus, it is not surprising that abnormalities in activin receptor expression and/or signaling are associated with various human diseases $(4,5)$. Activin has immunomodulatory functions and can have pro- or antiinflammatory activities $(3,5,6)$. In skin, activin overexpression enhances carcinogenesis through generation of a pro-tumorigenic immune cell response (4). In addition, mice overexpressing activin in keratinocytes (K14-Activin tg) of the epidermis and hair follicles present abnormal keratinocyte differentiation in the tail skin, enhanced wound repair and increased populations of regulatory T-cells (Treg) (7-9). Further, activin induces Langerhans cell (LC) differentiation (10), and follistatin overexpression in the epidermis of transgenic mice leads to reduction of the LC population (11). Finally, activin induces mast cell maturation and migration (12). Thus, activin is linked to particular biological processes, known to be involved in atopy, which made us wonder whether it could exert an active role in atopic dermatitis (AD).

Atopic dermatitis is the most common inflammatory skin disease, affects $10-20 \%$ of children and $1-3 \%$ of adults, and predisposes to asthma and allergic rhinitis (13). AD origins involve genetic, immune, and environmental factors. Briefly, impaired barrier function, which in $50 \%$ of the cases is caused by profilaggrin $(F L G)$ gene mutations, favors the penetration of irritants and allergens through the epidermis. This leads in the predisposed host to activation of LC, and subsequently to an initial Th2 cell polarization induced by specific cytokines, such as thymic stromal lymphopoietin (TSLP) (14). Recently, activation of intraepithelial 
lymphocytes (dendritic epidermal T-cells in mice, DETCs) by antigens expressed on stressed keratinocytes has been shown to contribute to Th2 cell polarization (15). Migration of inflammatory cells to the site of lesion and specific cytokine accumulation result in a Th2 to Th1 switch and the "chronic" phase of AD, characterized by keratinocyte hyperproliferation and epidermal thickening with prominent infiltrates of LC, eosinophils, monocyte-macrophages, and mast cells (16).

There is no current information about activin involvement in human skin allergic diseases. However, this cytokine is strongly associated with allergic asthma, one of the atopic diseases (17). Airway epithelial barrier presents several common functional features and immune reactivity with epidermal barrier. Therefore, we undertook an in vivo study which investigates the potential role of activin in a mouse model for allergic dermatitis $(18,19)$. Repeated epicutaneous sensitization with ovalbumin (OVA) leads to AD-like lesions in mice characterized by progressive thickening of dermis and epidermis, and the presence of inflammatory infiltrates in the skin. Further, epicutaneously OVA-treated mice develop an early Th2-polarization, characterized by increased mRNA levels of IL4, IL13, and IL5, and high levels of IgE and OVA-specific IgE, which is another common feature of acute human AD. After 3 weeks of OVA exposure, increased mRNA levels of IFN $\gamma$, IL12p35, and OVA-specific IgG2a are observed, which is reminiscent of the Th2 (acute phase) to Th1 (chronic phase) switch of human $\operatorname{AD}(18,20)$.

Ovalbumin sensitization of transgenic mice overexpressing activin in keratinocytes led to Th2-independent inflammation. Our data reveal that activin plays a protective role against antigenspecific dermatitis and suggest that it is involved in the onset of AD-like symptoms in an epicutaneous sensitization mouse model.

\section{MATERIALS AND METHODS}

\section{MICE - OVA-EPICUTANEOUS SENSITIZATION}

Wild-type (WT) CD1 (Crl:, Charles River, France), and K14Activin transgenic mice (K14-Act mice) (2, 4) were housed, fed, and bred under SPF (Specific Pathogenic Free) conditions, according to federal guidelines and the federal and local authorities approved procedures. Breeding was performed between WT female and K14-Act tg male mice. WT and K14-Act tg female littermates were used for the experiments. Four to six-week-old female mice were treated according to the OVA-epicutaneous sensitization protocol described previously (18). Briefly, at day 0 (d0), mice were anesthetized, shaved and tape-stripped 12 times. A $1 \mathrm{~cm}^{2}$ piece of sterile gauze containing $100 \mu \mathrm{l}$ of OVA (Sigma, Switzerland) $(1 \mathrm{mg} / \mathrm{ml}$ in $\mathrm{NaCl} 0.9 \%)$ or $100 \mu \mathrm{l}$ of $\mathrm{NaCl} 0.9 \%$ only (control - Ctl) was secured on the back with a bandage, left for 7 days, then removed, this process followed by a 14-day rest period. Epicutaneous sensitization with OVA was repeated three times. The mice were sacrificed on day 50 (d50), and skin biopsies and serum were collected. Four groups of mice [WT - vehicletreated (Ctl), WT OVA-treated, K14-Act vehicle-treated (Ctl), and K14-Act OVA-treated], each including 10-15 mice, were used.

\section{RNA EXTRACTION AND REAL-TIME RT-PCR}

Total RNA from dorsal skin mouse biopsies was extracted using the RNeasy Fibrous Tissue Mini Kit (Qiagen, Germany). RNA integrity was verified on an agarose gel under denaturating conditions. RNA ( $2 \mu \mathrm{g})$ was reverse-transcribed into cDNA using MMLV-reverse transcriptase (New England Biolabs, UK) as follows: $10 \mathrm{~min}$ at $25^{\circ} \mathrm{C}, 60 \mathrm{~min}$ at $42^{\circ} \mathrm{C}, 5 \mathrm{~min}$ at $95^{\circ} \mathrm{C}$. Real-time PCR analysis was performed on a StepOne ${ }^{\mathrm{TM}} \mathrm{PCR}$ apparatus (Applied Biosystems, UK) using a Power SYBR Green Master Mix (Applied Biosystems, UK) and specific primers. Samples were amplified as described (21). The primers were designed using the Roche software (Universal Probe Library, Assay Design Center) unless described differently (Table 1). Analysis of relative gene expression was performed using the $2^{-\Delta \Delta C T}$ method (22). Hypoxanthine guanine phosphoribosyl transferase (Hprt) mRNA was used as internal control. Statistical analysis was performed with the Mann-Whitney $U$-test.

\section{HISTOLOGY}

Dorsal skin biopsies were fixed overnight in freshly prepared PFA $4 \%$ (paraformaldehyde) at $4^{\circ} \mathrm{C}$, then washed in TBS and embedded in paraffin. Skin specimens of $5 \mu \mathrm{m}$ thickness were deparaffinized, rehydrated, and stained with hematoxylin and eosin as described (21). Stained sections were analyzed under the light microscope (Nikon Ellipse E400, Switzerland) coupled to a CCD camera.

\section{IMMUNOFLUORESCENCE}

Dorsal skin biopsies were snap frozen in isopentane, embedded in OCT and cut in $5 \mu \mathrm{m}$ cryosections. Specimens were fixed in ice-cold acetone, blocked with 5\% NGS (normal goat serum) TBS - GBA (glycine - BSA) for $1 \mathrm{~h}$ and incubated at room temperature (RT) with the primary antibody $-\gamma \delta$ TCR-FITC antibody (BD Biosciences San Diego, CA), anti-MPO (myeloperoxidase) (Neomarkers, Thermo Scientific, Fremont - CA, USA), anti-F4/80 (Caltag Invitrogen AG, Camarillo, CA, USA), or anti-langerin (CD207, clone 929F3.01, Dendritics, France). Three hours later, slides were rinsed and incubated for $1 \mathrm{~h}$ with the appropriate secondary antibody (Alexa Fluor 488 IgG, Molecular Probes, Invitrogen, Netherlands), when needed, then counterstained with DAPI and mounted with a fluorescent mounting medium (Dako, Denmark). The images were captured by a confocal microscope (LSM 700 Zeiss, Switzerland) and analyzed using the ZEN2010 software.

$\gamma \delta$ TCR-, MPO-, F4/80-, or langerin-positive cells were counted in $10-15$ microscope fields $(\times 20)$ per animal (five mice per group), and expressed as cells per millimeter of basal layer or per square millimeter of skin. Numbers in vehicle-treated WT animals were used as reference. Mean and SD were calculated and statistically significant differences were calculated with Mann-Whitney $U$-test.

\section{ELISA}

Detection of total IgE in serum was performed using a mouse IgE ELISA Set (BD OptEIA, BD Biosciences Pharmingen, Belgium). For OVA-specific IgE, a 96-well ELISA plate was coated with OVA $(40 \mu \mathrm{g} / \mathrm{ml})$ in sodium carbonate coating buffer overnight at $4^{\circ} \mathrm{C}$. After blocking with $10 \%$ heat inactivated FBS, mouse serum samples (diluted 1:20 for IgE) were added and incubated for $3 \mathrm{~h}$ at RT. Biotinylated detection antibodies (rat anti-mouse IgE - clone R35118 , BD Pharmingen, Belgium) and a streptavidin-HRP reagent were used for detection.

Total serum Ig2a levels were detected using a sandwich ELISA: briefly, the plate was coated with a capture antibody (rat 
Table 1 | Real-time PCR primers used in this study.

\begin{tabular}{llll}
\hline Target gene & Forward & Reverse & GenBank accession number \\
\|\| $1 \beta$ & TGAAGTTGACGGACCCCAAA & TGATGTGCTGCTGCGAGATT & NM_008361 \\
Tnf $\alpha$ & CCAGGCGGTGCCTATGTCT & GGCCATTTGGGACTTCTCAT & NM_013693 \\
Tslp & TGTGCCATTTCCTGAGTACCGTCA & NM_021367 \\
$\| 4$ & TCCTATCCCTGGCTGCCCTTCA & CGAGCTCACTCTCTGTGGTG & NM_021283 \\
$\| 13$ & CATCGGCATTTTGAACGAG & CGTTGCACAGGGGAGTCT & NM_008355 \\
$\| \mathrm{fn} \gamma$ & CCTCTGACCCTTAAGGAGCTTAT & TTCAAGACTTCAAAGAGTCTGAGGTA & NM_010548.1 \\
$\| 10$ & ATCTGGAGGAACTGGCAAAA & GTCCAGCTGGTCCTTTGTT & NM_010552.3 \\
Foxp3 & CAGAGCCACATGCTCCTAGA & GTGCAGCCCACACCCACCAG & NM_054039.1 \\
Rae-1 & ACCCTGGACTCTCCACCGCA & GCTACGATGCAGCAAGAGC & NM_020030 \\
Hprt & AGAAGCTGGGAGCTATGCAG & CCCAGGTGGCACTAGGAGT & NM_013556 \\
\hline
\end{tabular}

anti-mouse IgG2a - clone R11-89, BD Pharmingen) overnight at $4^{\circ} \mathrm{C}$, and serum samples were diluted 1:400 and incubated for $2 \mathrm{~h}$ at RT. A biotinylated antibody (Rat anti-mouse IgG2a - clone R1915, BD Pharmingen) and streptavidin-HRP reagent were used for detection.

Colorimetric reactions occurred with TMB substrate solution (Invitrogen, Netherlands) after $30 \mathrm{~min}$ incubation in the dark. Absorbance was measured at $450 \mathrm{~nm}$ corrected for absorbance at $620 \mathrm{~nm}$. Statistical analysis was performed with the MannWhitney $U$-test.

\section{RESULTS \\ OVA-EPICUTANEOUS SENSITIZATION OF K14-ACTIVIN $\operatorname{tg}$ MICE LEADS TO INFLAMMATION AND EPIDERMAL THICKENING}

After treatment of WT and K14-Act mice with either OVA or vehicle, histological analysis showed important differences between the four groups of mice, in terms of epidermis thickening and immune cell populations (Figures 1 and 2). In WT OVA-treated mice, the epidermis was thickened (2-fold) compared to WT vehicle-treated mice, and displayed lymphocytic exocytosis and parakeratotic hyperkeratosis (Figures 1A,B). Further, the dermis from WT OVA-treated mice showed features of fibrosis, as well as moderate infiltrates of lymphocytes, macrophages, and to a lesser extent neutrophils (Figures 2A,B,D). Untreated back skin from K14-Act mice is phenotypically normal (4), whereas vehicletreated skin from K14-Act mice was characterized by thickened epidermis (2-fold) and dermis compared to vehicle-treated WT mice (Figures 1A,B). Further, the epidermis of K14-Act OVAtreated mice was thickened compared to vehicle-treated WT mice (approximately 3-fold), and lymphocytic exocytosis and hyperparakeratosis were observed (Figures 1A,B). Inflammatory infiltrates, essentially composed of neutrophils, were more abundant in the dermis of K14-Act OVA-treated mice compared to the rest of the groups (Figure 1A; Figures 2A,D). Numbers of LC and Foxp3 mRNA, as an indicator of the Foxp3+ Treg cell population in skin, were not altered at the end of the protocol in any group (Figures 2C,D, and data not shown).

The expression of IL1 $\beta$ and TNF $\alpha$ was analyzed in order to further characterize the local inflammatory response. In addition, we analyzed the expression of TSLP, a marker cytokine for impaired barrier function and inducer of Th2-polarization. No differences in Il1 $\beta$, Tnfa, or Tslp mRNA levels were observed between untreated WT and K14-Act mice (Figures 3C,D, and data not shown). However, $I l 1 \beta$ and $T s l p$ mRNAs were significantly increased in vehicle-treated K14-Act mice as compared to vehicletreated WT mice (Figures 3A,B). No changes on Tnfa mRNA were observed between these two groups (data not shown). Obviously, the enhanced expression of pro-inflammatory cytokines results from the experimental protocol, including shaving, tape-stripping, and bandage application, which induces a non-specific inflammatory response to mechanical barrier injury and vehicle application. $I l 1 \beta$ and Tslp mRNAs were increased in the OVA-treated WT mice compared to the vehicle-treated WT group (Figures 3A,B). Tnfa mRNA was also mildly increased in the OVA-treated WT mice compared to the saline-treated WT animals, but there was no other significant difference when compared to other groups (data not shown). Inflammation in vehicle- and OVA-treated transgenic mice was associated with high local mRNA expression levels of both $I l 1 \beta$ and Tslp mRNAs, when compared to vehicle-treated WT group (Figures 3A,B). However, while antigen application did provoke a strong Il1 $\beta$ mRNA increase in K14-Act mice compared to the saline-treated K14-Act, Tslp mRNA was already elevated without OVA, and no further increase was noted by allergen sensitization (Figures 3A,B). These results reveal that repeated vehicle and OVA application provoked a differential cutaneous reaction, which was translated into a divergent cytokine expression pattern. Further, our data imply that activin overexpressing animals were more susceptible to inflammation after mechanical stress.

\section{REDUCED Th2-POLARIZATION IN K14-ACTIVIN MICE}

Acute AD involves a hypersensitivity response associated with Th2-polarization. Surprisingly, Il13 and Il4 mRNAs remained unchanged in the K14-Act OVA-treated mice compared to vehicletreated WT and K14-Act mice. This is an activin-dependent effect because the mRNAs of the same cytokines were increased in WT OVA-treated mouse skin, as expected. These results suggest that activin prevents a Th2-response to OVA stimulation (Figures 4A,B).

If $n \gamma$ mRNA expression, as a marker of Th1 response in skin appearing in chronic AD stages, was similar for all groups of animals (Figure 4C) Consequently, these mice did not show signs of chronic eczematous lesions during the experimental protocol. 

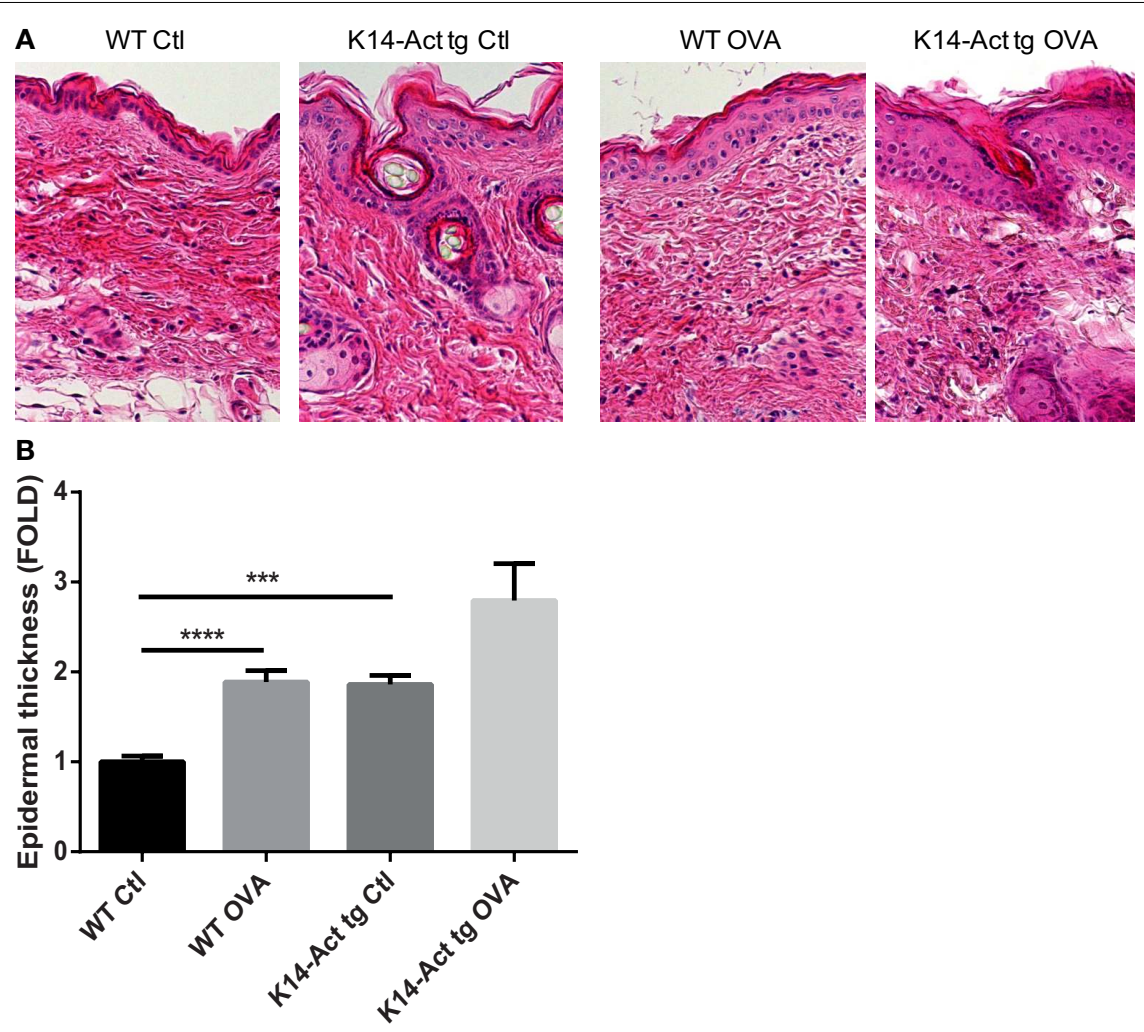

FIGURE 1 | OVA-epicutaneous sensitization leads to epidermal thickening and inflammation. (A) WT and K14-Act mice were treated with vehicle (Ctl) or OVA. Skin biopsies were stained for H\&E (20x objective). (B) Epidermal thickness (7-10 animals per group, five to seven fields per animal) in micrometer was measured on H\&E-stained biopsies from the four experimental groups using a scaled ocular lens on a light microscope. Mean values are represented as fold of the mean value of WT vehicle group $(\mathrm{Ctl}) \pm$ SEM. Statistical differences were calculated with the Mann-Whitney $U$-test $\left(p<0.05^{*}\right.$, $p<0.001^{* * *}$ ).

\section{ACTIVIN OVEREXPRESSION HAMPERS THE PRODUCTION OF IgES}

To better dissect the involvement of activin in the OVAepicutaneous sensitization, serum IgEs and IgG2a levels were measured. Serum IgEs were significantly increased only in the WT OVA-treated mice, whereas they remained at the basal level for the other three groups (Figure 5A). The discrete and non-significant increase in the vehicle-treated and OVA-treated K14-Act mice in comparison to the vehicle-treated WT animals indicates a role of mechanical barrier disruption in atopic sensitization.

Ovalbumin-specific IgEs were increased only in the WT-OVA mice group, and barely detectable in OVA-treated transgenic mice (Figure 5B). Thus, activin inhibits the antigen-specific immune response in this experimental setting.

Further, a minor increase was observed in total serum IgG2a, for the OVA-treated K14-Act group compared to the K14-Act Ctl mice, although OVA-specific IgG2a were not detectable (Figure 5C and data not shown). Thus, activin is involved in non-specific polyclonal IgG2a modulation, without inducing a Th1-polarization, which should be associated with higher levels of antigen-specific IgG2a and increased concentrations of Ifn $\gamma$. In sum, activin overexpression hampers the immune response by blocking IgE production.

\section{DEPLETION OF $\gamma \delta$ T-CELLS FROM K14-ACT MOUSE EPIDERMIS AFTER OVA SENSITIZATION}

$\gamma \delta$ T-cells are involved in the induction of Th2-response and $\operatorname{IgE}$ production after tissue damage (23). To test whether activin may involve $\gamma \delta$ T-cell mediated immune surveillance in this experimental setting, $\gamma \delta$ T-cells were quantified. A strong reduction of the epidermal $\gamma \delta$ T-cell population was noted in K14-Act animals, particularly after OVA treatment (Figure 6A). Further, mRNA levels of Rae1 (Retinoic acid early 1), an NKG2D ligand, were found significantly decreased in the K14-Act OVA group when compared to the WT OVA mice (Figure 6B). Given that epidermal $\gamma \delta \mathrm{T}$ cell numbers in untreated K14-Act mice as compared with WT mice are unaffected (4), our results suggest that activin obstructs maintenance of $\gamma \delta$ T-cells in hyperproliferative epidermis after mechanical injury, and this is further enhanced by antigen exposure. This significant depletion of the $\gamma \delta$ T-cell population in the K14-Act mice after epicutaneous sensitization may contribute to the reduced allergic reaction.

\section{UPREGULATION OF II10 AND I/17 mRNA EXPRESSION IN OVA-TREATED TRANSGENIC MICE}

IL10's main role is to suppress allergic responses, but it can also be a Th2-effector cytokine, according to the molecular context (24, 


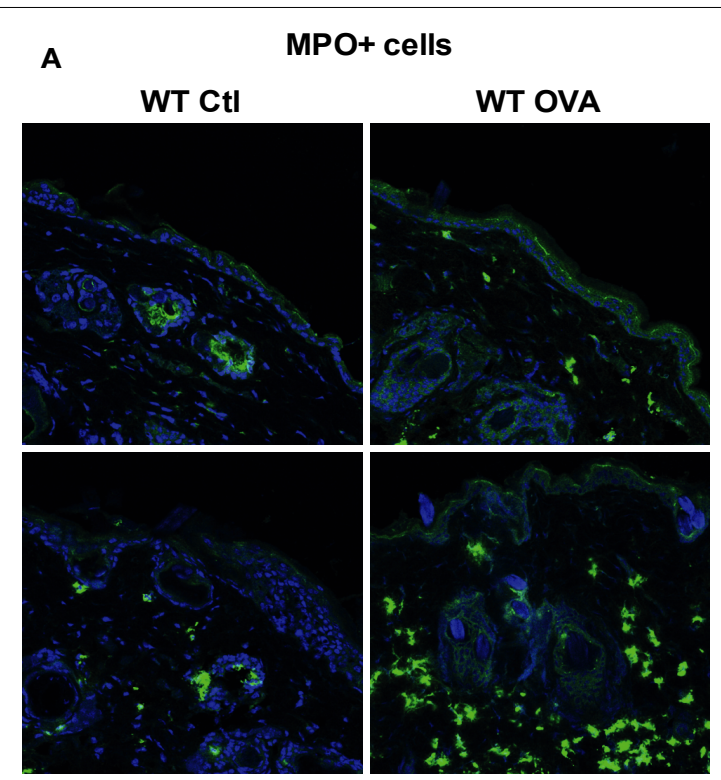

K14-Act tg CtI
K14-Act tg OVA
B
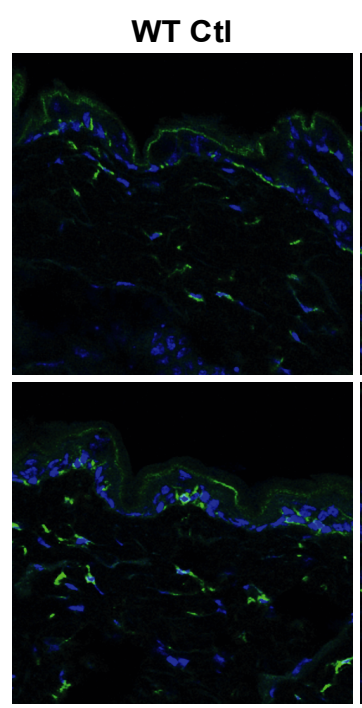

K14-Act tg CtI
F4/80+ cells

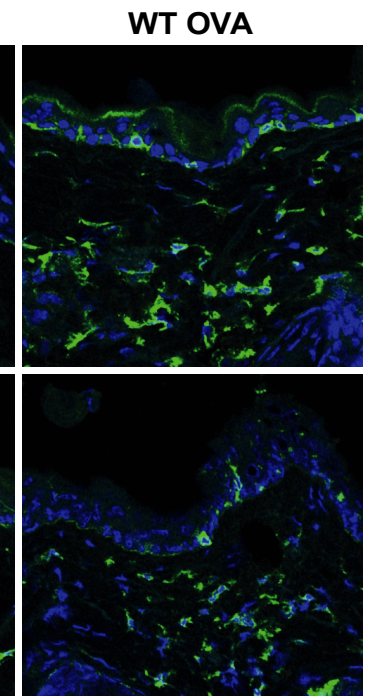

K14-Act tg OVA
C
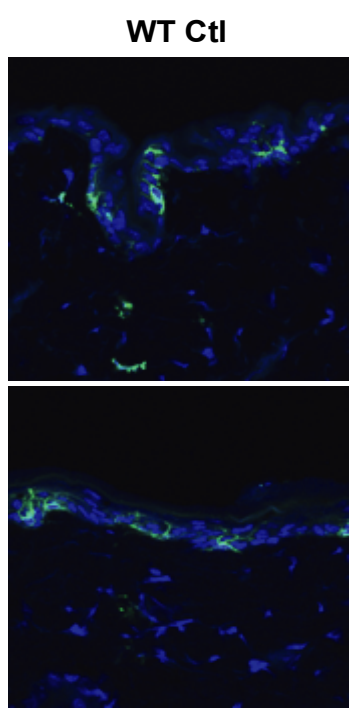

K14-Act tg CtI
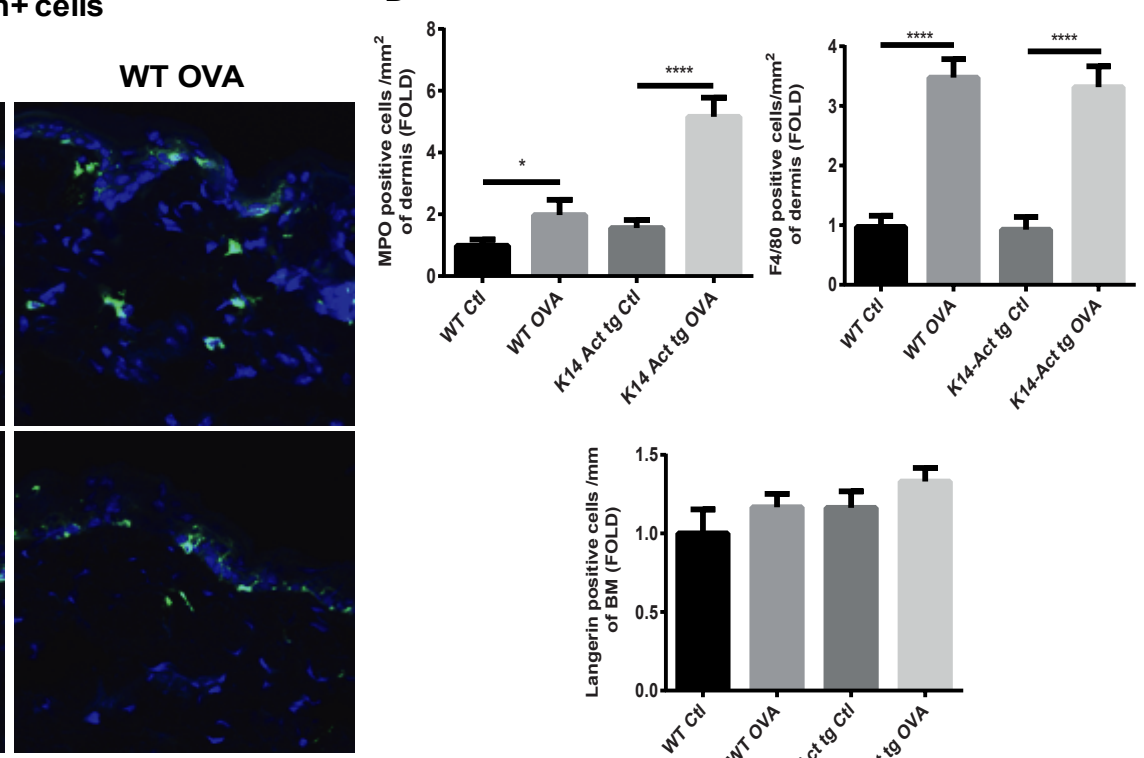

K14-Act tg OVA

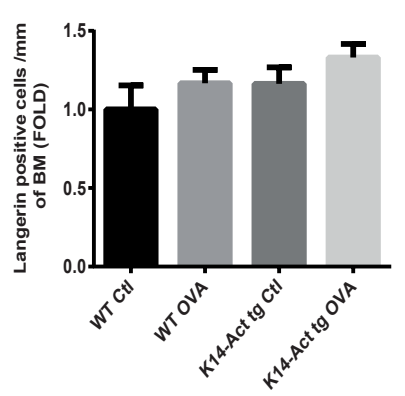

FIGURE 2 | Morphological and cell analysis of vehicle-treated and OVA-treated mice. Cryosections from the four experimental groups were obtained. $\mathrm{MPO}+(\mathbf{A}), \mathrm{F} 4 / 80+\mathbf{( B )}$, and langherin+ cells (C) were labeled with the respective antibodies, and analyzed with a confocal microscope (20 $\times$ objective). (D) MPO, F4/80, or langerin-positive cells were quantified and statistic analysis was performed with the Mann-Whitney $U$-test $\left(p<0.05^{*}\right.$ and $\left.p<0.001^{* *}\right)$.

25). Its expression was evaluated in dorsal skin biopsies obtained from vehicle or OVA-treated mice, by real-time PCR. Interestingly, Il10 mRNA expression was increased in OVA-treated K14-Act mice compared to OVA-treated WT mice (Figure 7A). IL10 secretion in the absence of Th2 cytokines, as for the OVA K14-Act mice (Figure 4), strongly suggests a regulatory function, which could explain the suppression of Th2-response and IgE production. IL10
mRNA expression is also increased in OVA-treated WT mice when compared to Ctl WT mice; in this case, IL10 could be co-secreted from Th2-cells (Figure 7A).

IL17A, mainly produced by Th17 cells but also by neutrophils and other immune cells, was measured as a marker of tissue inflammation and eventual TSLP-induced Th2-polarization inhibitor (26). Il17A mRNA was significantly increased in OVA-treated 

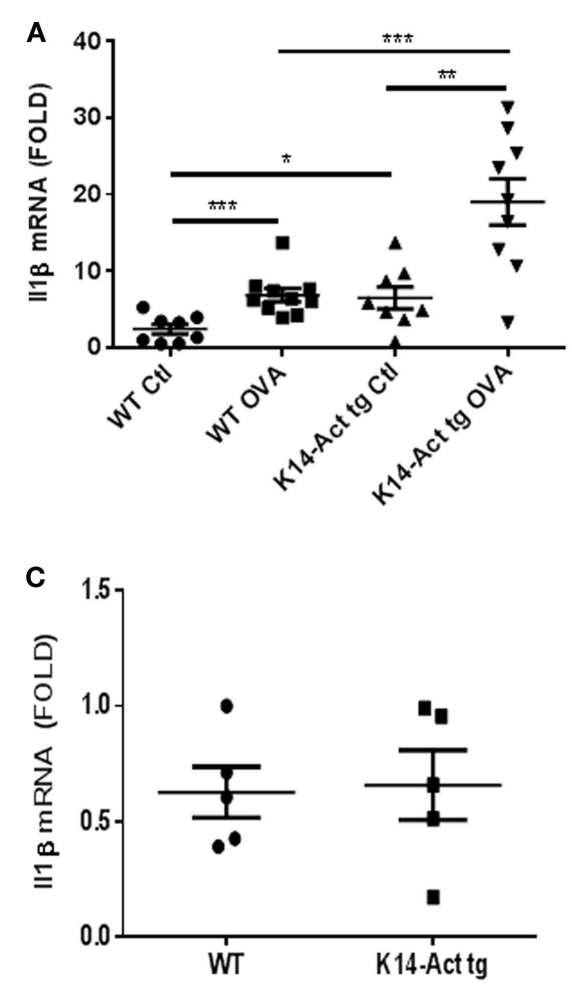

FIGURE 3 | Expression of pro-inflammatory cytokines in vehicle and OVA-treated mice. cDNAs were generated from WT and K14-Act mice and real-time PCR was performed. $m / L 1 \beta(\mathbf{A}, \mathbf{C})$ and $m T s / p(\mathbf{B}, \mathbf{D})$ mRNAs were amplified using CDNAs from untreated WT and K14-Act mice (C,D) or treated with vehicle or OVA $\mathbf{( A , B ) . ~ C t ~ v a l u e s ~ w e r e ~ n o r m a l i z e d ~ t o ~ e x p r e s s i o n ~ l e v e l s ~ o f ~}$

K14-Act skin biopsies compared to OVA-treated WT samples, suggesting the involvement of Th17-related cytokines in allergen epicutaneous sensitization (Figure 7B).

\section{DISCUSSION}

In this study we demonstrated for the first time that epidermal keratinocyte-derived activin protects from antigen-specific $\mathrm{T}$-cell immune response in an epicutaneous sensitization murine model, because: (1) It inhibited Th2-polarization after antigen-induced allergic dermatitis, (2) It hampered IgE and antigen-specific IgE production in mouse serum, and (3) It led to decrease of $\gamma \delta$ T-cell population in injured skin. These findings are novel and they provide further evidence about the role of activin in skin, already known for its involvement in wound healing and, skin morphogenesis and tumorigenesis $(2,4,9,27)$.

Ovalbumin-epicutaneous sensitization induces a Th2-specific response characterized by high IgE production (19). This response evolves during the time of treatment, and depends on the quantity of the antigen absorbed $(19,20,28)$. Activin strongly blocked this response because in OVA sensitized K14-Act transgenic animals, Th2-cytokine expression levels remained low, and total IgE and antigen-specific IgE serum levels were not significantly changed compared to vehicle-treated mice. In contrast, antigen-treated WT mice developed high total and OVA-specific IgE responses,
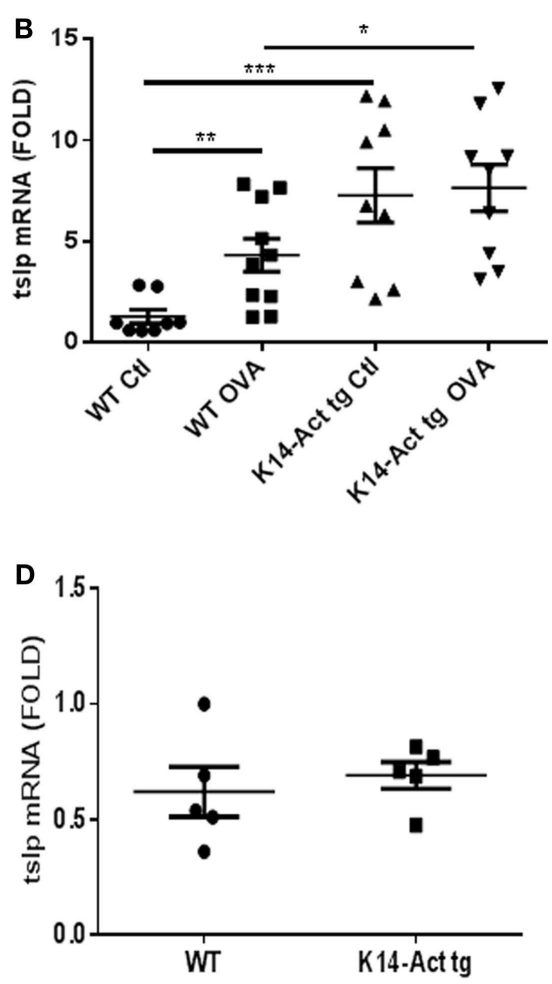

the hprt house-keeping gene. The experiment was realized in groups of five mice for the untreated and 8-10 mice for the vehicle- or OVA-treated mice. Results are presented in a scatter graph and mean values \pm SEM are shown. Statistical differences were calculated with the Mann-Whitney $U$-test $\left(p<0.05^{*}, p<0.01^{* *}\right.$, and $\left.p<0.001^{* * *}\right)$. associated with an increase in Th2-derived Il4 and Il13 mRNA expression, as expected.

The mechanism through which activin regulates antigen sensitization responses in skin is not elucidated. However, it is known that activin is involved in the antigen presentation process $(4,8$, $25,29,30$ ). Further, activin A suppresses effector Th2, but also Th1 immunity through generation of a specific subset of regulatory T-cells (25). Treg subsets regulate T-lymphocyte activation and suppress eosinophil and mast cell responses, inducing allergen peripheral tolerance and protecting from anaphylactic shock (31). Activin-induced Tregs in mouse lung draining lymph node (DLN) are CD4+CD25-Foxp3-, express IL10 and TGF 1 and inhibit CD11c+ DCs maturation $(7,8,25)$. Indeed, in our experimental setting, Il10 mRNA was increased in the OVA-treated K14-Act mice, which, in the absence of Th2-responses, may suggest the action of Treg cells. Except from Treg cells, IL10 is also secreted by macrophages, neutrophils, fibroblasts, and keratinocytes, together with other cytokines, such as TNF $\alpha$, IL1 $\beta$, IL8, and IL6, as a consequence of DNA damage or inflammation (32-34). However, the specific cytokine and cell type profile detected in this study strongly supports the immunosuppressive role of IL10 in these experimental conditions. The above data suggest that activin inhibits Th2-polarization in our model, through a Treg-dependent mechanism. The absence of a local Th1-polarization, in our study, does 


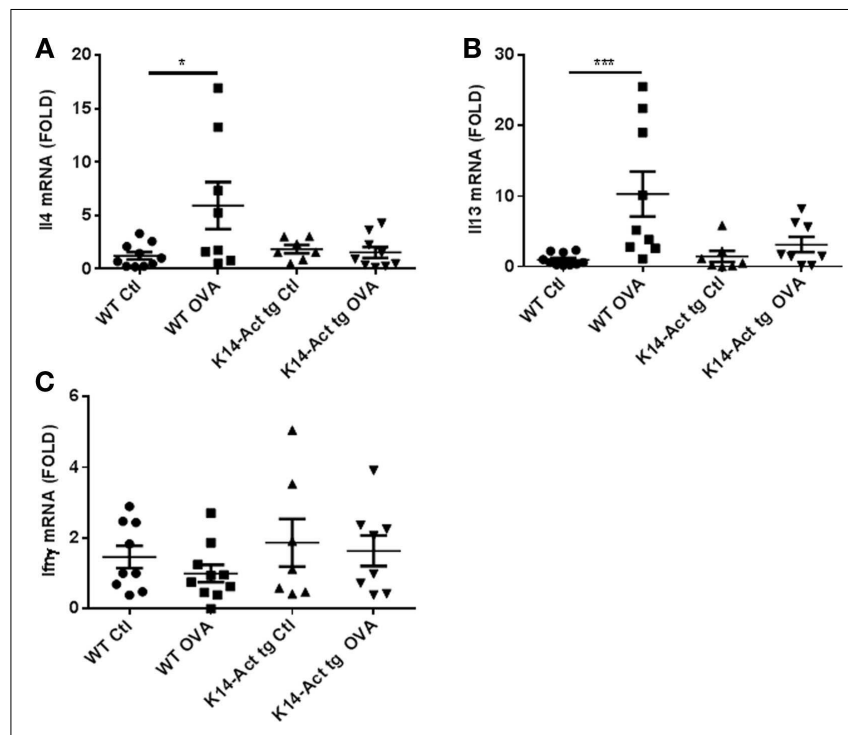

FIGURE 4 |Th2-specific cytokines increase upon OVA treatment of WT mice but not of K14-Act mice. Skin biopsies from WT and K14-Act mice, untreated or treated (vehicle-Ctl or OVA) were collected and used for RNA isolation and subsequent generation of cDNA. Real-time PCRs were performed using primers specific for (A) $m / 113$, (B) $m / / 4$, and (C) $m / f n \gamma$. Ct values were normalized to expression levels of the hprt house-keeping gene. The experiment was realized in groups of 8-10 mice. Results are presented in a scatter graph and mean values \pm SEM are shown.

Statistically significant differences were calculated with the Mann-Whitney U-test $\left(p<0.05^{*}, p<0.01^{* *}\right.$, and $\left.p<0.001^{* * *}\right)$. not exclude a broader activin-mediated suppressive effect on T-cell responses.

This is the first report providing data about the function of activin in antigen-induced cutaneous allergic reaction, which are consistent with its protective role from atopic pulmonary disease $(17,25)$. Activin is increased in serum and in CD4+ T-cells of patients with mild asthma, although there are no differences between healthy subjects and severely affected patients $(25,35)$. It is mainly secreted by immune, lung smooth muscle and epithelial cells and enhances epithelial and alveolar cell proliferation, promoting tissue remodeling after allergen exposure (35-38). In parallel, it is involved in the maturation of DCs and diminishes their antigen presenting capacity to T-cells (30), indicating that activin suppresses allergic reactions and maintains tissue homeostasis.

Barrier disruption-induced Th2-polarization is often preceded by increase of TSLP expression $(28,39)$. TSLP is an epithelialderived cytokine and signals through a heteromeric receptor (TSLPR and IL7r $\alpha$ ). It is involved in the activation and/or proliferation of several immune cell populations, such as $\mathrm{B}$ and $\mathrm{T}$ lymphocytes, basophils and eosinophils, and is essential for DCdriven Th2-polarization after mechanical injury of the epidermal barrier $(28,40,41)$. TSLP gene transcription is activated by IL1 $\beta$ and $\mathrm{TNF} \alpha$ (42). In our experimental setting, Tslp mRNA expression was increased in all three experimental groups, compared to WT Ctl mice, independently on the presence or not of antigen. TSLP upregulation is likely to be independent on the IL1 $\beta$ increase

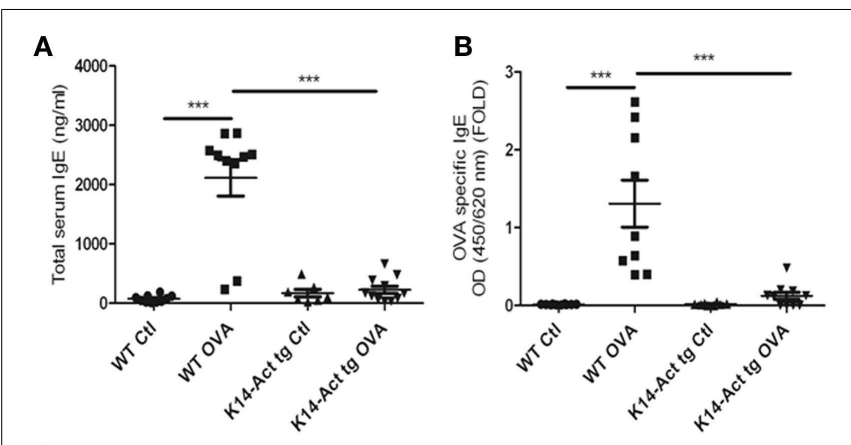

C

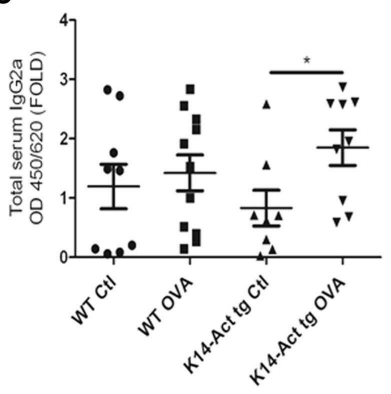

FIGURE $\mathbf{5}$ | Activin overexpression inhibits the production of IgEs after OVA treatment. WT and K14-Act mice treated with OVA or vehicle were sacrificed, and serum was collected. Total IgE (A), OVA-specific lgE (B), and total IgG2a (C) ELISA were performed as described in Section "Materials and Methods." The data are presented as a scatter plot and mean values are shown $(n=8-12)$. Statistically significant differences were calculated with the Mann-Whitney $U$-test $\left(p<0.05^{*}\right.$ and $\left.p<0.01^{* *}\right)$.

during epicutaneous sensitization, because the expression levels of their mRNAs did not correlate in the different experimental groups (Figures 3A,B). Indeed, Oyoshi et al. (28), showed a rapid and transient accumulation of TSLP in skin after tape-stripping. This suggests that different mechanisms are at work in our model in order to maintain TSLP expression in skin in a chronic manner.

These observations lead to the conclusion that activin plays a role downstream of TSLP and prevents Th2-cytokine expression. Bogiatzi et al. (26) recently showed that TSLP's capacity to induce a Th2-response depends on the cytokine milieu, and is inhibited when Th17-related cytokines are present. In line with this finding, IL17, IL1 $\beta$, and Smad signaling are positively modulated in OVAtreated K14-Act tg mice in our experimental setting, suggesting that activin intervenes during early immune responses.

Significant reduction of $\gamma \delta$ T-cell population in vehicle and OVA-treated K14-Act skin, compared to WT Ctl mice, is another feature of the activin-induced Th2 allergic reaction inhibition. Indeed, $\gamma \delta$ T-cells were shown to be required for the upregulation of Th2-derived cytokines after epicutaneous antigen sensitization, and they dramatically modulate the production of total and antigen-specific IgE (23). This link between lymphoid stress surveillance and atopic response, is further associated to Rae-1, a NKG2D membrane receptor ligand, which promotes $\gamma \delta$ T-cell activation (23). $\gamma \delta$ T-cells or DETCs reside in the murine epidermis and are activated after epithelial stress. They induce the elimination of damaged keratinocytes and are involved in the 


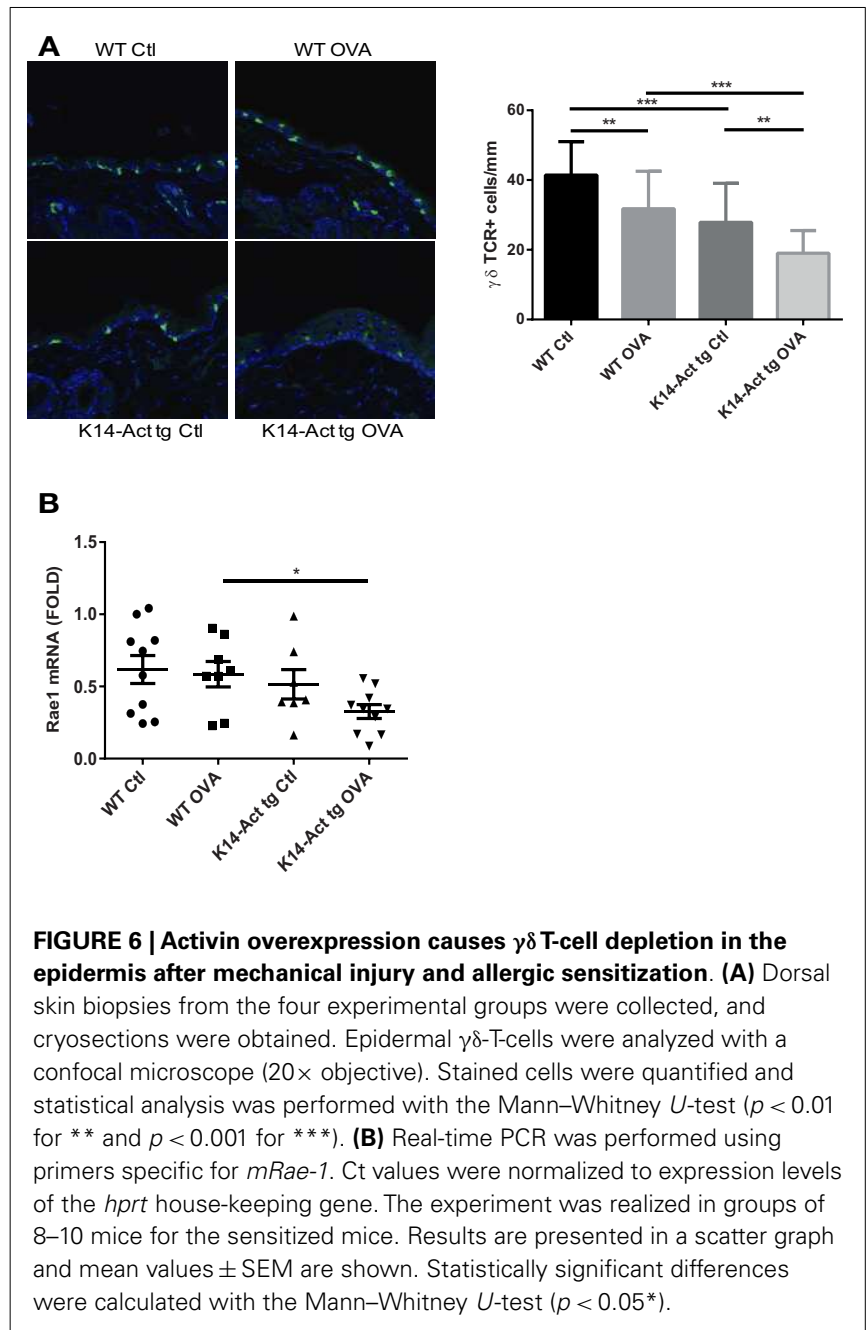

activation of adaptive immunity $(43,44)$. Intraepidermal $\gamma \delta \mathrm{T}$ cells in human skin contribute to efficient wound repair, although they are more scarce compared to mouse skin (45). The decrease of intraepidermal $\gamma \delta$ T-cells in vehicle-treated K14-Act mice, and most importantly in the OVA-treated group is consistent with reduced expression levels of Rae-1 mRNA. Thus, activin may not only affect $\gamma \delta$ T-cells, but also the expression of the activating ligand on keratinocytes. Conversely, the $\gamma \delta$ T-cell population (and Rae-1 expression) in WT mice, was not affected. Although it was shown that activin impairs human NKT cell functions without significantly modulating NKG2D receptor expression, little is known about the effect of activin on $\gamma \delta$ T-cell receptors (29). We showed that $\gamma \delta$ T-cells express activin receptors, but we could not detect increased apoptosis of these cells in the presence of high levels of activin during DMBA-TPA tumorigenesis. Rather, proliferation of $\gamma \delta \mathrm{T}$-cells was inhibited under these conditions (4). We propose that activin also attenuates $\gamma \delta$ T-cell function after tape-stripping, probably by inhibiting their proliferation, thereby leading to their depletion.

In this study, we also demonstrate the pro-inflammatory role of activin after epithelial barrier injury. Indeed, Il1 $\beta$ mRNA

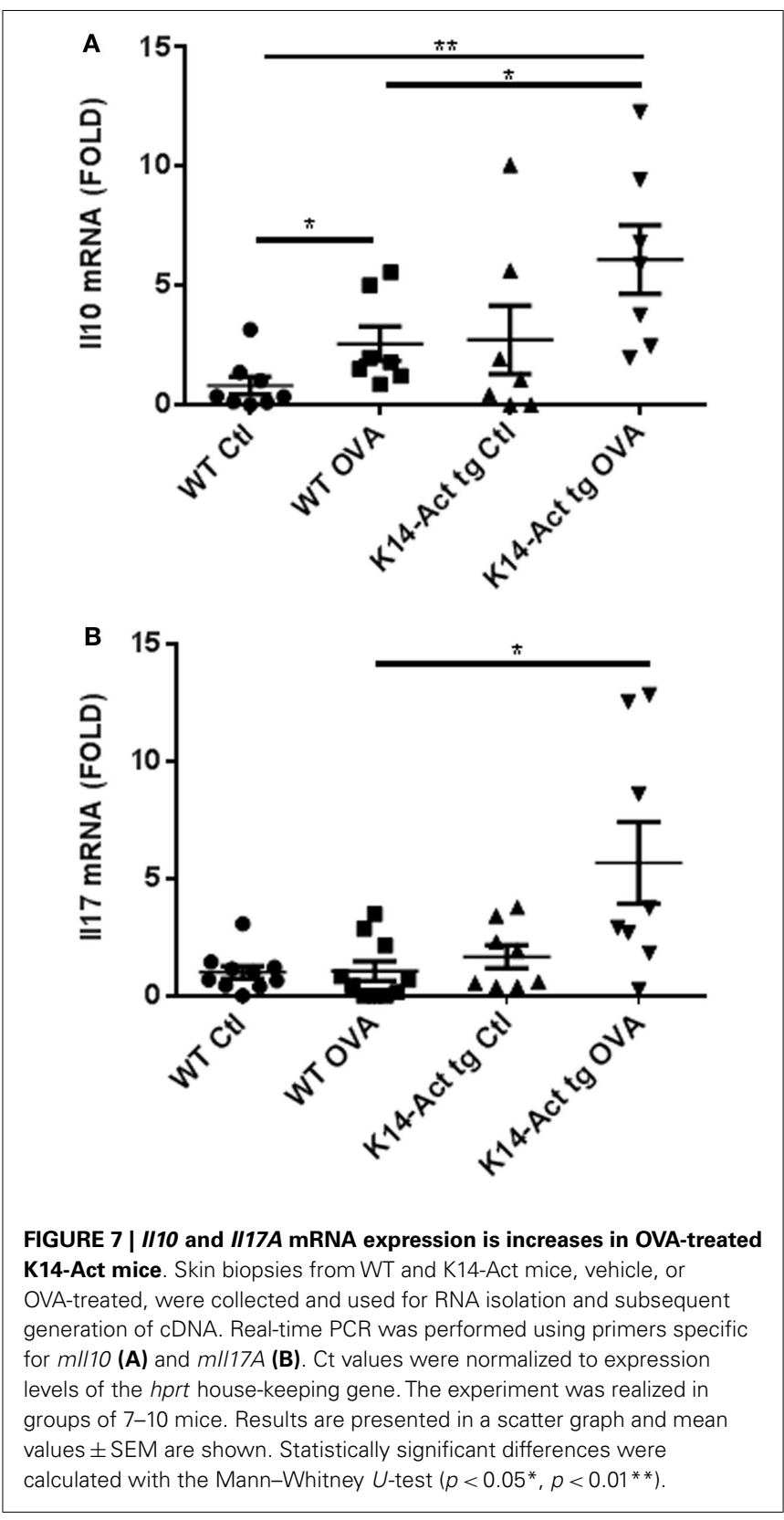

levels were increased in OVA-treated mice, and correlated with macrophage accumulation at the site of the treatment. This was consistent with previous reports demonstrating that $I l 1 \beta$ and Tnfa are the first cytokines induced after mechanical injury and epicutaneous sensitization $(20,28,46)$. In fact, activin overexpression triggers an inflammatory response in the mice treated only with saline, suggesting that they are more susceptible to mechanical disruption of the epidermal barrier. Indeed, activin $\mathrm{A}$ is a component of the innate immune response and triggers pro-inflammatory cytokine secretion in experimental inflammation (47). Immune responses involve very diverse and complex molecular mechanisms and cell types, and depend on the nature of "aggression," in order to protect the organism. Activin plays a central role in defense and is controlled by a well-defined cell 
and cytokine milieu (5). Accordingly, activin A's pro-inflammatory effect is dose and anatomical site-dependent $(25,48)$ and linked to the genetic background. CD1 K14-Act mice present spontaneous eye inflammation and periocular skin lesions at 5-6 months after birth. When backcrossed into an inbred background, such as $\mathrm{BALB} / \mathrm{c}$, the inflammatory lesions emerged earlier and also appeared at other body sites, and these manifestations were accelerated with generations. Skin biopsies from the affected regions of BALB/c K14-Act mice showed macrophage and neutrophil accumulation, but absence of eosinophils ( $\mathrm{MK}$ and $\mathrm{DH}$ unpublished observations).

In sum, we showed that activin protects from antigen-induced dermatitis in skin through modulation of immune responses after epicutaneous sensitization. Our results suggest that activin intervenes in at least two ways: (a) To favor secretion of proinflammatory cytokines after mechanical barrier disruption, and (b) to inhibit Th2-polarization and IgE generation. Further experiments in our model, including short OVA-epicutaneous sensitization followed by restimulation of DLN cells with OVA ex vivo, may allow to further dissect the mechanism through which activin

\section{REFERENCES}

1. Harrison CA, Gray PC, Vale WW, Robertson DM. Antagonists of activin signaling: mechanisms and potential biological applications. Trends Endocrinol Metab (2005) 16:73-8. doi:10.1016/j.tem.2005.01. 003

2. Munz B, Smola H, Engelhardt F, Bleuel K, Brauchle M, Lein I, et al. Overexpression of activin $\mathrm{A}$ in the skin of transgenic mice reveals new activities of activin in epidermal morphogenesis, dermal fibrosis and wound repair. EMBO J (1999) 18:5205-15. doi:10.1093/emboj/18. 19.5205

3. Antsiferova M, Werner S. The bright and the dark sides of activin in wound healing and cancer. J Cell Sci (2012) 125:3929-37. doi:10.1242/ jcs.094789

4. Antsiferova M, Huber M, Meyer M, Piwko-Czuchra A, Ramadan $\mathrm{T}$, MacLeod AS, et al. Activin enhances skin tumourigenesis and malignant progression by inducing a pro-tumourigenic immune cell response. Nat Commun (2011) 2:576. doi:10.1038/ncomms 1585

5. Werner S, Alzheimer C. Roles of activin in tissue repair, fibrosis, and inflammatory disease. Cytokine Growth Factor Rev (2006) 17:157-71. doi:10.1016/j.cytogfr.2006.01.001

6. Hedger MP, Winnall WR, Phillips DJ, de Kretser DM. The regulation and functions of activin and follistatin in inflammation and immunity. Vitam Horm (2011)
85:255-97. doi:10.1016/B978-012-385961-7.00013-5

7. Huber S, Schramm C. Role of activin $\mathrm{A}$ in the induction of Foxp3+ and Foxp3- CD4+ regulatory T cells. Crit Rev Immunol (2011) 31:53-60. doi:10.1615/ CritRevImmunol.v31.i1.50

8. Huber S, Stahl FR, Schrader J, Luth S, Presser K, Carambia A, et al. Activin a promotes the TGF-beta-induced conversion of CD4+CD25- T cells into Foxp3+ induced regulatory $\mathrm{T}$ cells. $J$ Immunol (2009) 182:4633-40. doi: 10.4049/jimmunol.0803143

9. Munz B, Hubner G, Tretter Y, Alzheimer C, Werner S. A novel role of activin in inflammation and repair. JEndocrinol (1999) 161:18793. doi:10.1677/joe.0.1610187

10. Musso T, Scutera S, Vermi W, Daniele R, Fornaro M, Castagnoli $\mathrm{C}$, et al. Activin A induces Langerhans cell differentiation in vitro and in human skin explants. PLoS ONE (2008) 3:e3271. doi:10.1371/ journal.pone.0003271

11. Stoitzner P, Stossel H, Wankell M, Hofer S, Heufler C, Werner S, et al. Langerhans cells are strongly reduced in the skin of transgenic mice overexpressing follistatin in the epidermis. Eur J Cell Biol (2005) 84:733-41. doi:10.1016/j.ejcb.2005. 04.003

12. Funaba M, Ikeda T, Ogawa K, Murakami M, Abe M. Role of activin A in murine mast cells: modulation of cell growth, differentiation, and migration. J Leukoc Biol

prevents antigen-induced immune responses in skin, and to focus on early time points.

Activin bioavailability and activity are tightly controlled by a complex network of secreted and intracellular molecules. The signals initiated by activin reach gene targets through a sophisticated pathway also firmly regulated at different levels, from the membrane to the nuclear level. Exhaustive analyses dissecting this molecular network in skin are necessary in order to consider activin or downstream mediators as successful candidates in the field of treatment for atopic diseases, ruling out activin's profibrotic, pro-inflammatory, and in particular its pro-tumorigenic effects.

\section{ACKNOWLEDGMENTS}

This work has been supported by the Swiss National Science Foundation (\# 31003A-120343) and the "Gottfried und Julia BangerterRhyner-Stiftung." We would like to thank M. Daniel Bachmann for skillful technical assistance. We would like to thank Dr. Maria Antsiferova, and Dr. Loredana Frasca for helpful discussions and critical reading of the paper.

(2003) 73:793-801. doi:10.1189/jlb. 0103012

13. Irvine AD, McLean WH, Leung DY. Filaggrin mutations associated with skin and allergic diseases. $N$ Engl J Med (2011) 365:1315-27. doi:10. 1056/NEJMra1011040

14. Leung DY, Boguniewicz M, Howell MD, Nomura I, Hamid QA. New insights into atopic dermatitis. J Clin Invest (2004) 113:651-7. doi: 10.1172/JCI200421060

15. Strid J, Tigelaar RE, Hayday AC. Skin immune surveillance by $\mathrm{T}$ cells - a new order? Semin Immunol (2009) 21:110-20. doi:10.1016/j. smim.2009.03.002

16. Bieber T. Atopic dermatitis. Ann Dermatol (2010) 22:125-37. doi:10. 5021/ad

17. Kariyawasam HH, Semitekolou M, Robinson DS, Xanthou G. ActivinA: a novel critical regulator of allergic asthma. Clin Exp Allergy (2011) 41:1505-14. doi:10.1111/j. 1365-2222.2011.03784.x

18. Spergel JM, Mizoguchi E, Brewer JP, Martin TR, Bhan AK, Geha RS. Epicutaneous sensitization with protein antigen induces localized allergic dermatitis and hyperresponsiveness to methacholine after single exposure to aerosolized antigen in mice. J Clin Invest (1998) 101:161422. doi:10.1172/JCI1647

19. Wang LF, Lin JY, Hsieh KH, Lin RH. Epicutaneous exposure of protein antigen induces a predominant Th2-like response with high IgE production in mice. J Immunol (1996) 156:4077-82.
20. Wang G, Savinko T, Wolff H, DieuNosjean MC, Kemeny L, Homey B, et al. Repeated epicutaneous exposures to ovalbumin progressively induce atopic dermatitis-like skin lesions in mice. Clin Exp Allergy (2007) 37:151-61. doi:10. 1111/j.1365-2222.2006.02621.x

21. Obarzanek-Fojt M, Favre B, Kypriotou M, Ryser S, Huber M, Hohl D. Homeodomain-only protein HOP is a novel modulator of late differentiation in keratinocytes. Eur J Cell Biol (2011) 90:279-90. doi:10.1016/ j.ejcb.2010.11.001

22. Livak KJ, Schmittgen TD. Analysis of relative gene expression data using real-time quantitative PCR and the 2(-Delta Delta C $(\mathrm{T})$ ) Method. Methods (2001) 25:402-8. doi:10.1006/meth.2001.1262

23. Strid J, Sobolev O, Zafirova B, Polic $\mathrm{B}$, Hayday A. The intraepithelial $\mathrm{T}$ cell response to NKG2D-ligands links lymphoid stress surveillance to atopy. Science (2011) 334:1293-7. doi:10.1126/science. 1211250

24. Mocellin S, Panelli MC, Wang E, Nagorsen D, Marincola FM. The dual role of IL-10. Trends Immunol (2003) 24:36-43. doi:10. 1016/S1471-4906(02)00009-1

25. Semitekolou M, Alissafi T, Aggelakopoulou M, Kourepini E, Kariyawasam $\mathrm{HH}$, Kay $\mathrm{AB}$, et al. Activin-A induces regulatory $\mathrm{T}$ cells that suppress $\mathrm{T}$ helper cell immune responses and protect from allergic airway disease. $J$ Exp Med (2009) 206:1769-85. doi:10.1084/jem.20082603 
26. Bogiatzi SI, Guillot-Delost M, Cappuccio A, Bichet JC, ChouchaneMlik O, Donnadieu $\mathrm{MH}$, et al. Multiple-checkpoint inhibition of thymic stromal lymphopoietininduced TH2 response by TH17related cytokines. J Allergy Clin Immunol (2012) 130(233-40):e5. doi:10.1016/j.jaci.2012.04.038

27. Bamberger C, Scharer A, Antsiferova $M$, Tychsen B, Pankow S, Muller M, et al. Activin controls skin morphogenesis and wound repair predominantly via stromal cells and in a concentration-dependent manner via keratinocytes. Am J Pathol (2005) 167:733-47. doi:10. 1016/S0002-9440(10)62047-0

28. Oyoshi MK, Larson RP, Ziegler SF, Geha RS. Mechanical injury polarizes skin dendritic cells to elicit a $\mathrm{T}(\mathrm{H}) 2$ response by inducing cutaneous thymic stromal lymphopoietin expression. J Allergy Clin Immunol (2010) 126:976-84, 984:e1-5. doi:10.1016/j.jaci.2010.08.041

29. Robson NC, Wei H, McAlpine T, Kirkpatrick N, Cebon J, Maraskovsky E. Activin-A attenuates several human natural killer cell functions. Blood (2009) 113:321825. doi:10.1182/blood-2008-07166926

30. Segerer SE, Muller N, Brandt J, Kapp M, Dietl J, Reichardt HM, et al. The glycoprotein-hormones activin A and inhibin A interfere with dendritic cell maturation. Reprod Biol Endocrinol (2008) 6:17. doi:10. 1186/1477-7827-6-17

31. Jutel M, Akdis CA. T-cell subset regulation in atopy. Curr Allergy Asthma Rep (2011) 11:139-45. doi: 10.1007/s11882-011-0178-7

32. Asadullah K, Sterry W, Volk HD. Interleukin-10 therapy - review of a new approach. Pharmacol Rev (2003) 55:241-69. doi:10.1124/pr. 55.2 .4

33. Glowacka E, Lewkowicz P, Rotsztejn H, Zalewska A. IL-8,
IL-12 and IL-10 cytokines generation by neutrophils, fibroblasts and neutrophils-fibroblasts interaction in psoriasis. $A d v$ Med Sci (2010) 55:254-60. doi:10.2478/v10039-010-0037-0

34. Monfrecola G, Gaudiello F, Cirillo T, Fabbrocini G, Balato A, Lembo S. Nicotinamide downregulates gene expression of interleukin-6, interleukin-10, monocyte chemoattractant protein-1, and tumour necrosis factor-alpha gene expression in HaCaT keratinocytes after ultraviolet B irradiation. Clin Exp Dermatol (2013) 38:185-8. doi:10.1111/ced.12018

35. Karagiannidis C, Hense G, Martin C, Epstein M, Ruckert B, Mantel PY, et al. Activin A is an acute allergenresponsive cytokine and provides a link to TGF-beta-mediated airway remodeling in asthma. $J$ Allergy Clin Immunol (2006) 117: 111-8. doi:10.1016/j.jaci.2005.09. 017

36. Hardy CL, Lemasurier JS, Olsson F, Dang $T$, Yao J, Yang $M$, et al. Interleukin-13 regulates secretion of the tumor growth factor\{beta\} superfamily cytokine activin $\mathrm{A}$ in allergic airway inflammation. Am J Respir Cell Mol Biol (2010) 42:667-75. doi:10.1165/rcmb.20080429OC

37. Hardy CL, Nguyen HA, Mohamud R, Yao J, Oh DY, Plebanski M, et al. The activin A antagonist follistatin inhibits asthmatic airway remodelling. Thorax (2013) 68: 9-18. doi:10.1136/thoraxjnl-2011201128

38. Ogawa K, Funaba M, Tsujimoto M. A dual role of activin $\mathrm{A}$ in regulating immunoglobulin production of $\mathrm{B}$ cells. J Leukoc Biol (2008) 83: 1451-8. doi:10.1189/jlb.1007710

39. Angelova-Fischer I, Fernandez IM, Donnadieu MH, Bulfone-Paus S, Zillikens D, Fischer TW, et al. Injury to the stratum corneum induces in vivo expression of human thymic stromal lymphopoietin in the epidermis. I Invest Dermato (2010) 130:2505-7. doi:10.1038/jid. 2010.143

40. Soumelis V, Reche PA, Kanzler H, Yuan W, Edward G, Homey B, et al. Human epithelial cells trigger dendritic cell mediated allergic inflammation by producing TSLP. Nat Immunol (2002) 3: 673-80.

41. Ziegler SF. The role of thymic stromal lymphopoietin (TSLP) in allergic disorders. Curr Opin Immunol (2010) 22:795-9. doi:10.1016/j.coi. 2010.10.020

42. Bogiatzi SI, Fernandez I, Bichet JC, Marloie-Provost MA, Volpe E, Sastre X, et al. Cutting edge: proinflammatory and Th2 cytokines synergize to induce thymic stromal lymphopoietin production by human skin keratinocytes. J Immunol (2007) 178:3373-7.

43. Jameson JM, Cauvi G, Witherden DA, Havran WL. A keratinocyteresponsive gamma delta TCR is necessary for dendritic epidermal $\mathrm{T}$ cell activation by damaged keratinocytes and maintenance in the epidermis. J Immunol (2004) 172:3573-9.

44. Shimura E, Hozumi N, Kanagawa $\mathrm{O}$, Metzger $\mathrm{D}$, Chambon $\mathrm{P}$, Radtke F, et al. Epidermal gammadelta $\mathrm{T}$ cells sense precancerous cellular dysregulation and initiate immune responses. Int Immunol (2010) 22:329-40. doi:10. 1093/intimm/dxq014

45. Toulon A, Breton L, Taylor KR, Tenenhaus M, Bhavsar D, Lanigan $\mathrm{C}$, et al. A role for human skin-resident $\mathrm{T}$ cells in wound healing. J Exp Med (2009) 206: 743-50. doi:10.1084/jem.20081787

46. Nickoloff BJ, Naidu Y. Perturbation of epidermal barrier function correlates with initiation of cytokine cascade in human skin. J Am Acad Dermatol (1994) 30:
535-46. doi:10.1016/S01909622(94)70059-1

47. Jones KL, Mansell A, Patella S, Scott BJ, Hedger MP, de Kretser DM, et al. Activin A is a critical component of the inflammatory response, and its binding protein, follistatin, reduces mortality in endotoxemia. Proc Natl Acad Sci U S A (2007) 104:16239_ 44. doi:10.1073/pnas.0705971104

48. Hardy CL, O'Connor AE, Yao J, Sebire K, de Kretser DM, Rolland JM, et al. Follistatin is a candidate endogenous negative regulator of activin A in experimental allergic asthma. Clin Exp Allergy (2006) 36:941-50. doi:10. 1111/j.1365-2222.2006.02523.x

Conflict of Interest Statement: The authors declare that the research was conducted in the absence of any commercial or financial relationships that could be construed as a potential conflict of interest.

Received: 04 May 2013; accepted: 07 August 2013; published online: 22 August 2013.

Citation: Kypriotou $M$, Rivero $D$, Haller S, Mariotto A, Huber $M$ Acha-Orbea H, Werner $S$ and Hohl $D$ (2013) Activin A inhibits antigeninduced allergy in murine epicutaneous sensitization. Front. Immunol. 4:246. doi: 10.3389/fimmu.2013.00246

This article was submitted to Inflammation, a section of the journal Frontiers in Immunology.

Copyright (c) 2013 Kypriotou, Rivero, Haller, Mariotto, Huber, Acha-Orbea, Werner and Hohl. This is an open-access article distributed under the terms of the Creative Commons Attribution License (CC BY). The use, distribution or reproduction in other forums is permitted, provided the original author(s) or licensor are credited and that the original publication in this journal is cited, in accordance with accepted academic practice. No use, distribution or reproduction is permitted which does not comply with these terms. 